\title{
Body waves in poroelastic media saturated by two immiscible fluids
}

\author{
Kagan Tuncay ${ }^{1}$ and M. Yavuz Corapcioglu \\ Department of Civil Engineering, Texas A\&M University, College Station
}

\begin{abstract}
A study of body waves in elastic porous media saturated by two immiscible Newtonianfluids is presented. We analytically show the existence of three compressional waves and one rotational wave in an infinite porous medium. The first and second compressional waves are analogousto the fast and slow compressional waves in Biot's theory. The third compressional wave is associated with the pressure difference between the fluid phases and dependent on the slope of capillary pressure-saturationrelation. Effect of a second fluid phase on the fast and slow waves is numerically investigatedfor Massillon sandstone saturated by air and water phases. A peak in the attenuation of the first and second compressional waves is observed at high water saturations. Both the first and second compressional waves exhibit a drop in the phase velocity in the presence of air. The results are compared with the experimental data available in the literature. Although the phase velocity of the first compressionaland rotational waves are well predicted by the theory, there is a discrepancy between the experimental and theoretical values of attenuationcoefficients. The causes of discrepancy are explained based on experimental observations of other researchers.
\end{abstract}

\section{Introduction}

Biot's theory of wave propagation in linear, elastic, saturated porous media has been the basis of many velocity and attenuation analyses [Biot, 1956a,b]. Biot demonstrated the presence of two compressional and one rotational wave in a porous medium saturated by a single fluid phase. The first compressional wave (also referred to as fast wave) is analogous to the compressional wave in elastic media. The second compressional wave is slower and highly attenuated. Because of its highly dissipative behavior, this wave is very difficult to observe. The second compressional wave was first observed by Plona [1980] (also see Berryman [1980]). However, we must note that neither of these waves propagate as a wave in the fluid or in the matrix alone, both travel jointly in the matrix and pore fluid. All waves are dispersed and attenuated. Attenuation arises because of the relative movement of solid and fluid phases. At low frequencies, attenuation is related to the permeability of solid matrix. Since laminar flow assumption does not hold for high frequencies, the theory needs modifications [Biot, 1956b]. We refer to Corapcioglu and Tuncay [1996] for an extensive review of Biot's theory.

In addition to the three types of body waves, other types of waves exist close to the interfaces. Analogous to elastic media, Deresiewicz [1962] and Jones [1961] showed the existence of surface waves in saturated porous media. They examined surface waves by considering the coupling of the transverse and the fast compressional waves. Later Tajuddin [1984] presented a study of Rayleigh waves considering all three types of body waves. Love waves which appear due to

'Now at Izmir Institute of Technology, Cankaya, Izmir, Turkey.

Copyright 1996 by the American Geophysical Union.

Paper number 96JB02297.

0148-0227/96/96JB-02297\$09.00 stratification of the Earth were investigated by Deresiewicz [1961, 1964, 1965] and Chattopadhyay and De [1983].

In contrast to porous media saturated by a single fluid, wave propagation in porous media saturated by multiphase fluids received limited attention from the researchers. The general trend is to extend Biot's formulation developed for saturated medium by replacing model parameters with the ones modified for the fluid-fluid or fluid-gas mixtures [Domenico, 1974; Mochizuki, 1982; Murphy, 1984; Pride et al., 1992]. This approach results in two compressional waves and has been shown to be successful in predicting the first compressional and rotational wave velocities for practical purposes. Brutsaert [1964] who extended Biot's theory appears to be the first one to predict three compressional waves. The third compressional wave arises due to presence of a second fluid phase in the pores. Brutsaert and Luthin [1964] provided experimental data which agrees with the results of Brutsaert's [1964] theory. The third compressional wave was also predicted by Garg and Nayfeh [1986] and Santos et al. [1990]. We should note that in these studies, although same type of governing equations were employed, the expressions for material constants differed. Miksis [1988] developed a model for wave attenuation in partially saturated rocks based on the local three-phase physics in the pore space. Tuncay [1995] derived the governing equations and constitutive relations of elastic porous media saturated by two Newtonian fluids by employing the volume-averaging technique.

In this study, starting from the governing equations, we investigate the types of waves and their characteristics in infinite isotropic porous media saturated by two fluids. We show that three compressional waves and one rotational wave exist. All waves are dispersed and attenuated. First and second compressional waves are similar to those in saturated media. We show that the third compressional wave occurs because of the existence of capillary pressure. Because of the very low velocity and high attenuation of the third compressional wave, 
an experimental observation does not appear to be possible. Finally, we compare the results of model with experimental data obtained in terms of phase velocities and attenuation coefficients of the first compressional and rotational waves.

\section{Final Set of Governing Equations}

We start by introducing the governing equations for elastic porous media saturated by two immiscible Newtonian fluids, for example, water and air. In the derivation, we assume that there is no mass exchange between the phases and the phases are at rest. Furthermore, the solid phase is assumed to be isotropic, experiencing small deformations and providing all shear resistance of the porous medium. Momentum transfer terms are expressed in terms of intrinsic and relative permeabilities assuming the validity of Darcy's law. At very low saturations (i.e., residual saturations), the theory may not be valid since not all phases of the medium are interconnected.

Furthermore, the theory employed in this study is limited to low-frequency wave propagation. Let $L, I$ and $r$ be the characteristic lengths of the macroscopic scale, averaging volume, and pore scale, respectively. The required condition for the volume averaging is $r \ll<\ll L$. In this study we assume that this requirement is satisfied, and furthermore, if $\lambda$ is the wavelength of the wave, we assume $l<<\lambda$. Therefore we limit the present study to low-frequency waves. In the case of two pore fluids, the low-frequency limit should be lower than that of a single fluid case since the size of averaging volume would be larger to include the presence of two pore fluids. In the case of a single pore fluid, the size of the averaging volume is determined by the pore structure only. However, when two fluids are present in the pores, the orientation of fluid phases is an important factor on the size of averaging volume.

In addition to assuming an isotropic elastic porous matrix saturated by two immiscible Newtonian fluids, validity of Darcy's Law, and low-frequency wave propagation, we furthermore assumed the validity of the relationship between capillary pressure and a fluid saturation, and negligibility of cross permeabilities known as the Yuster effect.

The governing equations are obtained in terms of displacements of solid and fluid phases as [Tuncay, 1995]

$$
\begin{aligned}
\left\langle\rho_{s}\right) \frac{\partial^{2} \bar{u}_{s}}{\partial t^{2}}-\nabla\left(\left(a_{11}+\frac{G_{f r}}{3}\right) \nabla \cdot \bar{u}_{s}+a_{12} \nabla \cdot \overline{u_{1}}+a_{13} \nabla \cdot \overline{u_{2}}\right) \\
+\nabla \cdot\left(G_{f r} \bar{\nabla} \overline{u_{s}}\right)+C_{1}\left(\overline{v_{1}}-\bar{v}\right)+C_{2}\left(\overline{v_{2}}-\overline{v_{s}}\right) \\
\left\langle\rho_{1}\right\rangle \frac{\partial^{2} \overline{u_{1}}}{\partial t^{2}}-\nabla\left(a_{21} \nabla \cdot \overline{u_{s}}+a_{22} \nabla \cdot \overline{u_{1}}+a_{23} \nabla \cdot \overline{u_{2}}\right)-C_{1}\left(\overline{v_{1}}-\overline{v_{s}}\right) \\
\left\langle\rho_{2}\right\rangle \frac{\partial^{2} \overline{u_{2}}}{\partial t^{2}}-\nabla\left(a_{31} \nabla \cdot \overline{u_{s}}+a_{32} \nabla \cdot \overline{u_{1}}+a_{33} \nabla \cdot \overline{u_{2}}\right)-C_{2}\left(\overline{v_{2}}-\overline{v_{s}}\right)
\end{aligned}
$$

where angle brackets and overbar are the volume average and intrinsic average operators, respectively. Subscripts s, 1 , and 2 correspond to the solid phase, nonwetting phase, and wetting phase, respectively. In (1)-(3), $p_{j}$ and $u_{i}$ are the density and displacement vector of phase $i$, respectively. $G_{f r}$ is the drained shear modulus of the solid matrix. The constants $a_{i j}$ are given by

$$
\begin{aligned}
a_{11} A_{3}-K_{s} & {\left[A_{1} \alpha_{s}\left(K_{1} A_{2} S_{1}+K_{2} K_{1}+K_{2} A_{2}\left(1-S_{1}\right)\right)\right.} \\
& \left.+K_{s} K_{f}\left(1-\alpha_{s}\right)\left(K_{1}\left(1-S_{1}\right)+S_{1} K_{2}+A_{2}\right)\right]
\end{aligned}
$$

$$
\begin{gathered}
a_{12} A_{3}-K_{1} K_{s} A_{1} S_{1}\left(1-\alpha_{s}\right)\left(A_{2}+K_{2}\right) ; \\
a_{13} A_{3}-K_{2} K_{s} A_{1}\left(1-S_{1}\right)\left(1-\alpha_{s}\right)\left(A_{2}+K_{1}\right) \\
a_{21}-a_{12} ; \\
a_{22} A_{3}-K_{1} S_{1}^{2}\left(1-\alpha_{s}\right)\left[K_{s}^{2}\left(1-\alpha_{s}\right)\left(K_{2}+A_{2} / S_{1}\right)\right. \\
\left.+K_{2} A_{2} A_{1}\left(1-S_{1}\right) / S_{1}\right] \\
a_{23} A_{3}=-K_{1} K_{2} S_{1}\left(1-S_{1}\right)\left(1-\alpha_{s}\right)\left[-K_{s}^{2}\left(1-\alpha_{s}\right)+A_{2} A_{1}\right] ; \\
a_{31}-a_{13} \\
a_{32}=a_{23} ; \\
a_{33} A_{3}=K_{2}\left(1-S_{1}\right)^{2}\left(1-\alpha_{s}\right)\left[K_{s}^{2}\left(1-\alpha_{s}\right)\left(K_{1}+A_{2} /\left(1-S_{1}\right)\right)\right. \\
\left.+K_{1} A_{2} A_{1} S_{1} /\left(1-S_{1}\right)\right]
\end{gathered}
$$

where

$$
\begin{gathered}
A_{1}-\alpha_{s} K_{s}-K_{f r} \quad ; \quad A_{2}=\frac{d P_{\text {cap }}}{d S_{1}} S_{1}\left(1-S_{1}\right) \\
A_{3}=A_{1}\left(K_{1} A_{2} S_{1}+K_{1} K_{2}+K_{2} A_{2}\left(1-S_{1}\right)\right) \\
+K_{s}^{2}\left(1-\alpha_{s}\right)\left(K_{1}\left(1-S_{1}\right)+A_{2}+K_{2} S_{1}\right)
\end{gathered}
$$

In (4)-(10), $K_{\mathrm{fr}}, K_{\mathrm{i}}, \alpha_{\mathrm{s}}, S_{1}$, and $P_{\text {cap }}$ are the drained bulk modulus of the solid matrix, bulk modulus of phase $i$, volume fraction of the solid phase, saturation of the nonwetting phase, and capillary pressure, respectively. $P_{\text {cap }}$, which is equal to the pressure difference between the nonwetting and wetting phases, is assumed to be a function of the saturation of the nonwetting phase, $S_{1}$ only. Assuming the validity of Darcy's law, $C_{1}$ and $C_{2}$ become

$$
\begin{gathered}
C_{1}-\frac{\left(1-\alpha_{s}\right)^{2} S_{1}^{2} \mu_{1}}{K k_{r l}} \\
C_{2}-\frac{\left(1-\alpha_{s}\right)^{2}\left(1-S_{1}\right)^{2} \mu_{2}}{K k_{r 2}}
\end{gathered}
$$

where $K, \mu_{i}, k_{r}$ are the intrinsic permeability, viscosity, and relative permeability of phase $\mathrm{i}$, respectively. Equations (1) and (3) reduce to Biots [1956a] equations for a single fluid phase by setting $S_{1}$ and $A_{2}$ to zero.

\section{Compressional Waves}

The formulation for the compressional waves is obtained by applying the divergence operator to (1)-(3),

$$
\begin{gathered}
\left\langle\rho_{s}\right\rangle \frac{\partial^{2} e_{s}}{\partial t^{2}}-a_{11}^{*} \nabla^{2} e_{s}+a_{12} \nabla^{2} e_{1}+a_{13} \nabla^{2} e_{2}+C_{1}\left(\frac{\partial e_{1}}{\partial t}-\frac{\partial e_{s}}{\partial t}\right) \\
+C_{2}\left(\frac{\partial e_{2}}{\partial t}-\frac{\partial e_{s}}{\partial t}\right)
\end{gathered}
$$

$\left\langle\rho_{1}\right) \frac{\partial^{2} \epsilon_{1}}{\partial t^{2}}-a_{21} \nabla^{2} e_{s}+a_{22} \nabla^{2} e_{1}+a_{23} \nabla^{2} e_{2}-C_{1}\left(\frac{\partial e_{1}}{\partial t}-\frac{\partial e_{s}}{\partial t}\right)$

$\left\langle\rho_{2}\right\rangle \frac{\partial^{2} e_{2}}{\partial t^{2}}-a_{31} \nabla^{2} e_{s}+a_{32} \nabla^{2} e_{1}+a_{33} \nabla^{2} e_{2}-C_{2}\left(\frac{\partial e_{2}}{\partial t}-\frac{\partial e_{s}}{\partial t}\right)$

where $\epsilon_{\mathrm{j}}=\nabla \cdot \bar{u}_{\mathrm{j}}$ and $\mathrm{a}_{11}{ }^{*}=a_{11}+4 \mathrm{G}_{\mathrm{f}} / 3$. The dilatational plane harmonic waves propagating along the $\mathrm{z}$ direction are given by

$$
\epsilon_{j}-B_{j} e^{(\xi z-\infty)}
$$


where $B_{j}$ is the wave amplitude, $\xi$ is the wave number, $\omega$ is the frequency, and $i$ is the imaginary number. In general, $\xi$ is a complex number. For convenience we rewrite (16) as

$$
\epsilon_{j}-B_{j} e^{-\varepsilon, z} e^{(\xi, z-\infty)}
$$

where $\xi_{i}$ and $\xi_{r}$ are the imaginary and real parts of $\boldsymbol{\xi}$, respectively. The imaginary part of the wave number $\xi_{i}$ is usually called the attenuation coefficient. The phase velocity is defined as $c=\omega / \xi_{\mathrm{r}}$. Substitution of (16) in (1)-(3) yields

$$
\begin{aligned}
& \left\{-\omega^{2}\left[\begin{array}{ccc}
\left\langle\rho_{s}\right\rangle & 0 & 0 \\
0 & \left\langle\rho_{1}\right\rangle & 0 \\
0 & 0 & \left\langle\rho_{2}\right\rangle
\end{array}\right]+\xi^{2}\left[\begin{array}{ccc}
a_{11} & a_{12} & a_{13} \\
a_{21} & a_{22} & a_{23} \\
a_{31} & a_{32} & a_{33}
\end{array}\right]\right. \\
& \left.+i \omega\left[\begin{array}{ccc}
-C_{1}-C_{2} & C_{1} & C_{2} \\
C_{1} & -C_{1} & 0 \\
C_{2} & 0 & -C_{2}
\end{array}\right]\right\}\left[\begin{array}{c}
B_{s} \\
B_{1} \\
B_{2}
\end{array}\right]-\left[\begin{array}{l}
0 \\
0 \\
0
\end{array}\right]
\end{aligned}
$$

which implies that for nonzero solutions the determinant of the coefficient matrix must be equal to zero. This equation is known as the dispersion equation in wave mechanics. The determinant can be expressed as

$$
Z_{1} X^{3}+Z_{2} X^{2}+Z_{3} X+Z_{4}=0
$$

where $X=\omega^{2} / \xi^{2}$. The complex coefficients of (19) are given by

$$
\begin{aligned}
& Z_{1}=\frac{C_{1} C_{2}\left(\left\langle\rho_{1}\right\rangle+\left\langle\rho_{2}\right\rangle+\left\langle\rho_{s}\right\rangle\right)-\left\langle\rho_{s}\right\rangle\left\langle\rho_{1}\right\rangle\left\langle\rho_{2}\right\rangle \omega^{2}}{\omega^{2}} \\
& -i \frac{C_{2}\left\langle\rho_{1}\right\rangle\left(\left\langle\rho_{2}\right\rangle+\left\langle\rho_{s}\right\rangle\right)+C_{1}\left\langle\rho_{2}\right\rangle\left\langle\left(\rho_{1}\right\rangle+\left\langle\rho_{s}\right\rangle\right)}{\omega} \\
& Z_{2}=-\frac{a_{11}\left(C_{1} C_{2}-\left\langle\rho_{1}\right\rangle\left\langle\rho_{2}\right\rangle \omega^{2}\right)+2 C_{1} C_{2}\left(a_{12}+a_{13}+a_{23}\right)}{\omega^{2}} \\
& -i \frac{a_{11}\left(C_{2}\left\langle\rho_{1}\right\rangle+C_{1}\left\langle\rho_{2}\right\rangle\right)+2 a_{12} C_{1}\left\langle\rho_{2}\right\rangle+2 a_{13} C_{2}\left\langle\rho_{1}\right\rangle}{\omega} \\
& -i \frac{a_{22}\left(C_{1} C_{2}-\left\langle\rho_{s}\right\rangle\left\langle\rho_{2}\right\rangle \omega^{2}\right)+a_{33}\left(C_{1} C_{2}-\left\langle\rho_{s}\right\rangle\left\langle\rho_{1}\right\rangle \omega^{2}\right)}{\omega^{2}} \\
& \left.+C_{2}\left(\left\langle\rho_{2}\right\rangle+\left\langle\rho_{s}\right\rangle\right)+C_{1}\left\langle\rho_{2}\right\rangle\right)+a_{33}\left(C_{2}\left\langle\rho_{1}\right\rangle+C_{1}\left(\left\langle\rho_{s}\right\rangle+\left\langle\rho_{1}\right\rangle\right)\right) \\
& \omega
\end{aligned}
$$

$$
\begin{gathered}
Z_{3}--a_{11}\left(a_{22}\left\langle p_{2}\right\rangle+a_{33}\left(\rho_{1}\right)\right)-a_{12}^{2}\left\langle\rho_{2}\right\rangle-a_{13}^{2}\left\langle\rho_{1}\right\rangle \\
+\left\langle p_{s}\right\rangle\left(a_{22} a_{33}-a_{23}^{2}\right)-i_{11}^{a_{11}\left(a_{22} C_{2}+a_{33} C_{1}\right)} \\
\omega \\
-i \frac{-a_{12}^{2} C_{2}-2 a_{12}\left(a_{23} C_{2}-a_{33} C_{1}\right)-a_{13}^{2} C_{1}}{\omega} \\
-i \frac{+2 a_{13}\left(a_{22} C_{2}-a_{23} C_{1}\right)+\left(C_{1}+C_{2}\right)\left(a_{22} a_{33}-a_{23}^{2}\right)}{\omega}
\end{gathered}
$$

$Z_{4}-a_{11}\left(a_{22} a_{33}-a_{23}^{2}\right)-a_{12}^{2} a_{33}+a_{13}\left(2 a_{12} a_{23}-a_{13} a_{22}\right)$
In general, for a given frequency $\omega$, the polynomial in (19) has three complex roots and the wave number $\xi$, has six roots. However, only three of these roots are physically possible, that is, the amplitudes of waves should decrease so that the imaginary part of $\xi$ must be greater than zero. This implies the existence of three compressional waves in a poroelastic medium saturated by two immiscible fluids. When we set $A_{2}=0$, we find $Z_{4}=0$ which indicates that one of the compressional waves is associated with the pressure difference between two fluid phases, that is, capillary pressure.

\section{Rotational Waves}

The formulation for the rotational waves is obtained by applying the curl operator to (1)-(3)

$$
\begin{aligned}
& \left\langle\boldsymbol{\rho}_{s}\right\rangle \frac{\partial^{2} \mathbf{Q}_{s}}{\partial t^{2}}-G_{f r} \nabla^{2} \mathbf{Q}_{s}+C_{1}\left(\frac{\partial \mathbf{Q}_{1}}{\partial t}-\frac{\partial \mathbf{Q}_{s}}{\partial t}\right)+C_{2}\left(\frac{\partial \mathbf{Q}_{2}}{\partial t}-\frac{\partial \mathbf{Q}_{s}}{\partial t}\right) \\
& \left\langle\boldsymbol{\rho}_{1}\right\rangle \frac{\partial^{2} \mathbf{a}_{1}}{\partial t^{2}}-C_{1}\left(\frac{\partial \mathbf{a}_{1}}{\partial t}-\frac{\partial \mathbf{a}_{s}}{\partial t}\right) \\
& \left\langle\boldsymbol{\rho}_{2}\right\rangle \frac{\partial^{2} \boldsymbol{Q}_{2}}{\partial t^{2}}=-C_{2}\left(\frac{\partial \mathbf{Q}_{2}}{\partial t}-\frac{\partial \mathbf{Q}_{s}}{\partial t}\right)
\end{aligned}
$$

where $\mathbf{Q}_{\mathrm{j}}=\nabla \times \overline{\mathbf{u}}_{\mathrm{j}}$. The substitution of harmonic waves as given by Eq. (16) in Eqs. (24)-(26) yields

$$
\begin{aligned}
& \left\{-\omega^{2}\left[\begin{array}{ccc}
\left\langle\rho_{s}\right\rangle & 0 & 0 \\
0 & \left\langle\rho_{1}\right\rangle & 0 \\
0 & 0 & \left\langle\rho_{2}\right\rangle
\end{array}\right]+\xi^{2}\left[\begin{array}{ccc}
G_{f r} & 0 & 0 \\
0 & 0 & 0 \\
0 & 0 & 0
\end{array}\right]\right. \\
& \left.+i \omega\left[\begin{array}{ccc}
-C_{1}-C_{2} & C_{1} & C_{2} \\
C_{1} & -C_{1} & 0 \\
C_{2} & 0 & -C_{2}
\end{array}\right]\right\}\left[\begin{array}{c}
B_{s} \\
B_{1} \\
B_{2}
\end{array}\right]-\left[\begin{array}{l}
0 \\
0 \\
0
\end{array}\right]
\end{aligned}
$$

The dispersion equation is the determinant of the coefficient matrix and is in the form of

$$
X^{2}\left(Z_{1} X+Z_{2}\right)=0
$$

where

$$
\begin{aligned}
& Z_{1}-\frac{C_{1} C_{2}\left(\left\langle p_{1}\right\rangle+\left\langle p_{2}\right\rangle+\left\langle p_{2}\right\rangle\right)-\left\langle p_{2}\right\rangle\left\langle p_{1}\right\rangle\left\langle p_{2}\right\rangle \omega^{2}}{\omega^{2}} \\
& -i \frac{\left.C_{2}\left(p_{1}\right\rangle\left\langle\left\langle p_{2}\right\rangle+\left\langle p_{s}\right\rangle\right)+C_{1}\left\langle p_{2}\right\rangle\right)\left(\left\langle p_{1}\right\rangle+\left\langle p_{s}\right\rangle\right)}{\omega} \\
& Z_{2}=-\frac{G_{r f}\left(C_{1} C_{2}-\left\langle\rho_{1}\right\rangle\left\langle\rho_{2}\right\rangle \omega^{2}\right)}{\omega^{2}}+i \frac{G_{t}\left(C_{2}\left\langle\rho_{1}\right\rangle+C_{1}\left\langle\rho_{2}\right\rangle\right)}{\omega}
\end{aligned}
$$

Equation (28) shows the existence of a single rotational wave in porous medium saturated by two immiscible fluids.

\section{Numerical Results}

We solve (19) and (28) for Massillon sandstone saturated by air and water. The material properties of Massillon 
Table 1. Material Parameters of Massillon Sandstone Saturated by Air and Water Phases

\begin{tabular}{|c|c|c|}
\hline Parameter & Symbol & Value \\
\hline Bulk modulus of solid matrix, GPa & $\mathbf{K}$ & 1.02 \\
\hline Bulk modulus of solid grains, GPa & & 35.00 \\
\hline Shear modulus of solid matrix, GPa & & 1.44 \\
\hline Density of solid grains, $\mathrm{kg} / \mathrm{m}^{3}$ & & 2650.00 \\
\hline Intrinsic permeability, $\mathbf{m}^{2}$ & $\mathbf{K}$ & $9 \times 10^{-13}$ \\
\hline Volume fraction of solid phase & $\boldsymbol{\alpha}_{\mathrm{s}}$ & 0.77 \\
\hline Density of water, $\mathrm{kg} / \mathrm{m}^{3}$ & $\mathbf{p}_{2}$ & 997.00 \\
\hline Bulk modulus of water, GPa & $\mathbf{K}$ & 2.25 \\
\hline Viscosity of water, $\mathrm{Pa}$ s & $\boldsymbol{\mu}_{2}^{2}$ & $1 \times 10^{-3}$ \\
\hline Density of air, $\mathrm{kg} / \mathrm{m}^{3}$ & $P_{1}$ & 1.10 \\
\hline Bulk modulus of air, $\mathrm{MPa}$ & $\mathbf{K}_{1}$ & 0.145 \\
\hline Viscosity of air, $\mathrm{Pa} \mathrm{s}$ & $\boldsymbol{\mu}_{1}$ & $18 \times 10^{-6}$ \\
\hline
\end{tabular}

After Murphy[1982, 1984]

sandstone taken from Murphy [1982, 1984] are listed in Table 1. Van Genuchter's [1980] closed form expressions for the capillary pressure-saturation relations are employed to obtain $\mathbf{d P}_{\text {cap }} / \mathrm{dS}_{1}$ in (9). Van Genuchten proposed that

$$
\frac{S_{2}-S_{r 2}}{S_{m 2}-S_{r 2}}-\left(1+\left(\frac{\alpha P_{c a p}}{100}\right)^{a}\right)^{-m}
$$

where $P_{\text {cap }}$ is the capillary pressure $\left(N / \mathrm{m}^{2}\right), S_{2}$ is the water saturation, $S_{n}$ is the irreducible water saturation, $S_{m 2}$ is the upper limit of water saturation in a two-phase medium, $m=1$ $1 / n$, and $\alpha$ and $n$ are material parameters. Parameters of (31) are chosen as $\alpha=0.025, n=10$, and $S_{n 2}=0.0$. We use the relative permeability curves of Wyckoff and Botset [1936] shown in Figure 1.

Figures 2-5 illustrate the effect of water saturation on the phase velocities at four different frequencies. From this point on we will represent the compressional waves by "P" and rotational wave by "S." Since there are three compressional waves, we will number them according to the magnitude of their phase velocity, Pl being the fastest. We observe a sudden drop in the phase velocity of the first compressional wave because of high compressibility of air (Figure 2). The reduc-

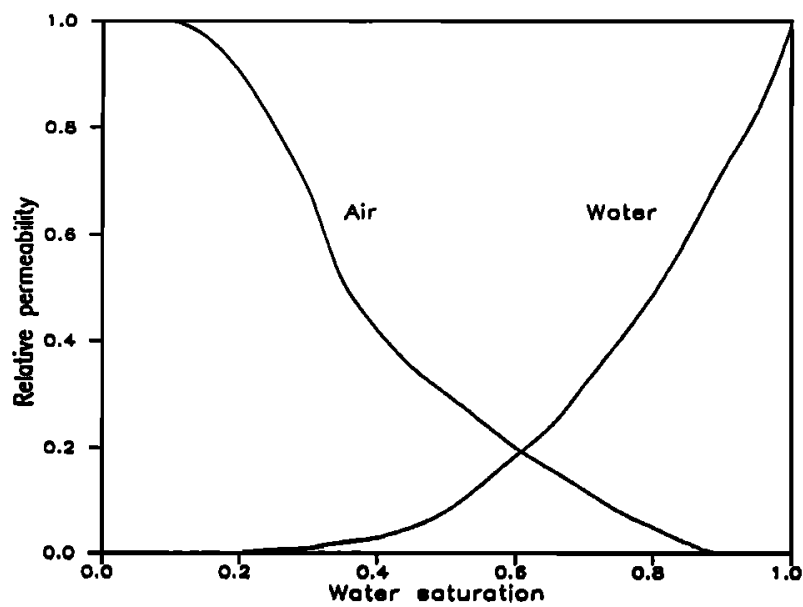

Figure 1. Relative permeability curves [after Wyckoff and Botset, 1936].

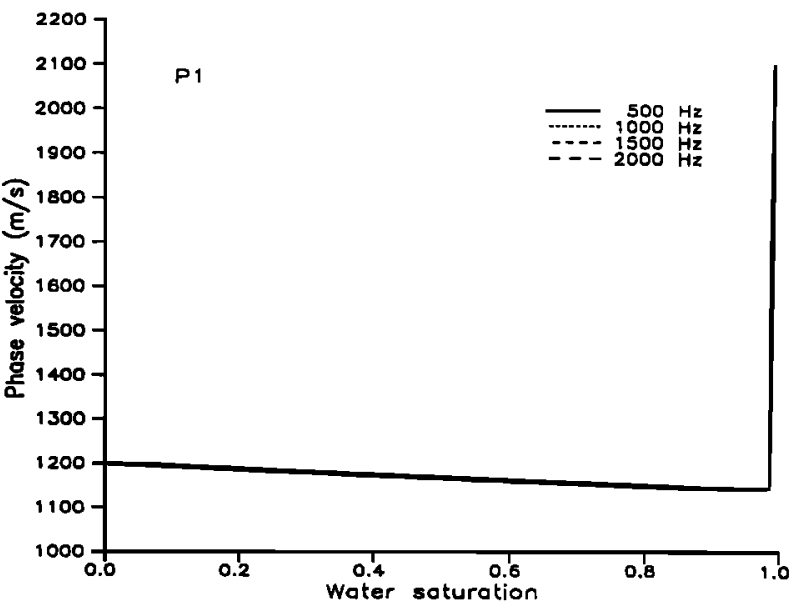

Figure 2. Effect of water saturation on the phase velocity of $\mathbf{P l}$.

tion in the phase velocity of $P 1$ because of $1 \%$ air is almost $50 \%$. We observe a similar trend in the phase velocity of the second compressional wave, P2 (Figure 3). The third compressional wave, which arises because of the pressure difference between the fluid phases, has the lowest phase velocity (Figure 4). As expected, P3 vanishes when only one fluid phase exists. P3's maximum velocity in the frequency range of interest is less than $1 \mathrm{~m} / \mathrm{s}$ which makes it very difficult to observe, if not impossible. Figure 5 illustrates the phase velocity of the rotational wave as a function of saturation. We note that the drop in the phase velocity is not observed in the rotational wave. The phase velocity of $\mathbf{S}$ shows linear dependence on water saturation. The jumps in P1 and P2 can be explained by the considerable changes in compressibility of pore fluids with a highly compressible phase, that is, air.

Figures 6-9 illustrate the frequency dependence of phase velocities of body waves. The drop in the phase velocity of $P 1$ can also be seen in Figure 6. Comparing Figures 6-8, we observe that the phase velocities of $\mathrm{P} 2$ and $\mathrm{P3}$ approach zero when the frequency approaches zero. Thus we can conclude that $P 1$ is a wave analogous to the compressional wave in elastic solids. Figure 8 shows that the maximum phase velocity of P3 is less than $6 \mathrm{~m} / \mathrm{s}$. Therefore, P1 and P2 correspond to

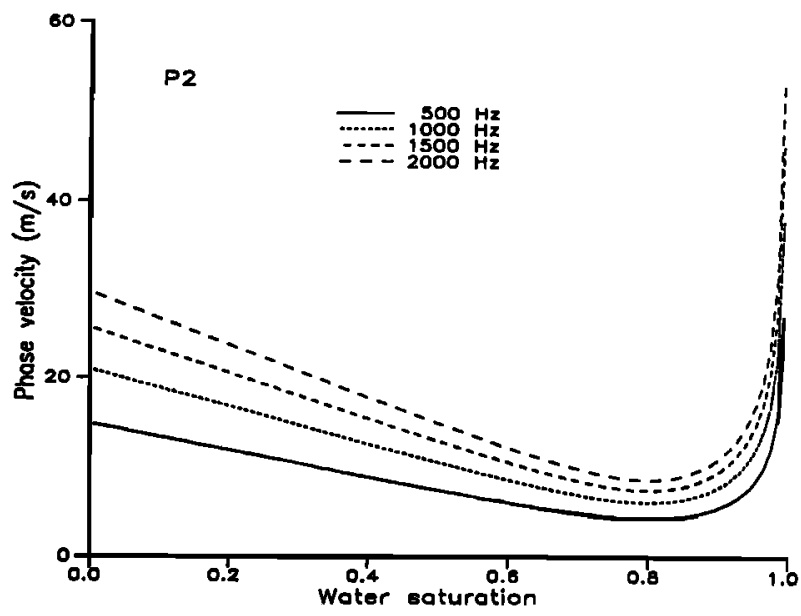

Figure 3. Effect of water saturation on the phase velocity of $\mathbf{P} 2$. 


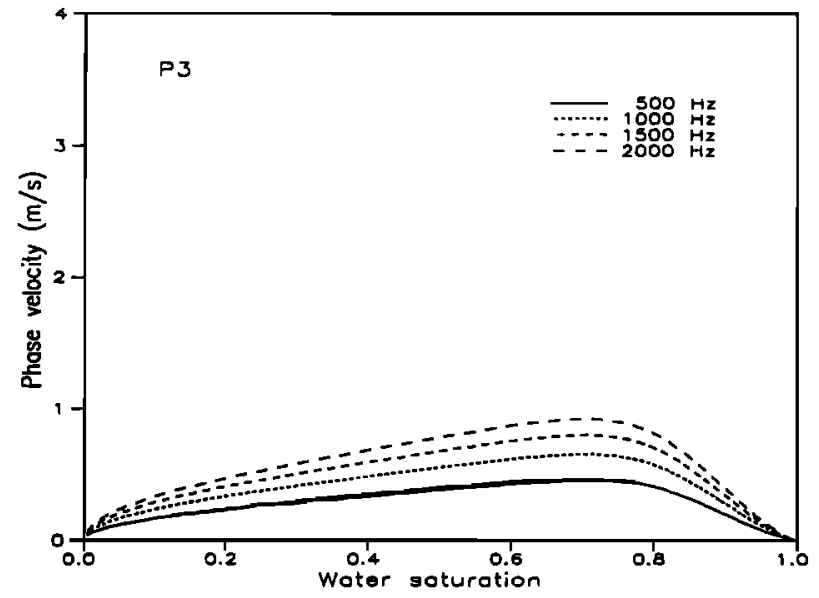

Figure 4. Effect of water saturation on the phase velocity of $\mathbf{P 3}$.

the fast and slow waves in Biot's theory. Figure 9 demonstrates the frequency dependence of the rotational wave. We observe that as the water saturation increases, the frequency dependence of phase velocity of $\mathbf{S}$ becomes more significant.

Figures 10-13 show the effect of water saturation on the attenuation coefficient, that is, the imaginary part of wave number. We should note that the attenuation coefficient calculated is the part due to the contribution of the relative movement between solid and fluid phases (equations (11)(12)). Inelastic energy losses are not taken into consideration. Figure 10 shows that the attenuation coefficient of P1 starts from zero and gradually increases as the water saturation increases. We observe a sudden drop when the water saturation approaches unity. We should note that viscosity of air is much smaller than that of water which explains why the first compressional wave is so little attenuated in air-saturated specimens. In case of a more viscous fluid than air, one should expect a curve starting from a nonzero attenuation coefficient. The attenuation coefficient of $\mathbf{P 2}$ as a function of water saturation is illustrated in Figure 11. Again, we observe a peak in the attenuation coefficient around $S_{2}=0.8$. We should note that the attenuation of the second compressional wave is determined by the kinematic viscosity, that is, the viscosity- to density ratio. The kinematic viscosity of air is much higher

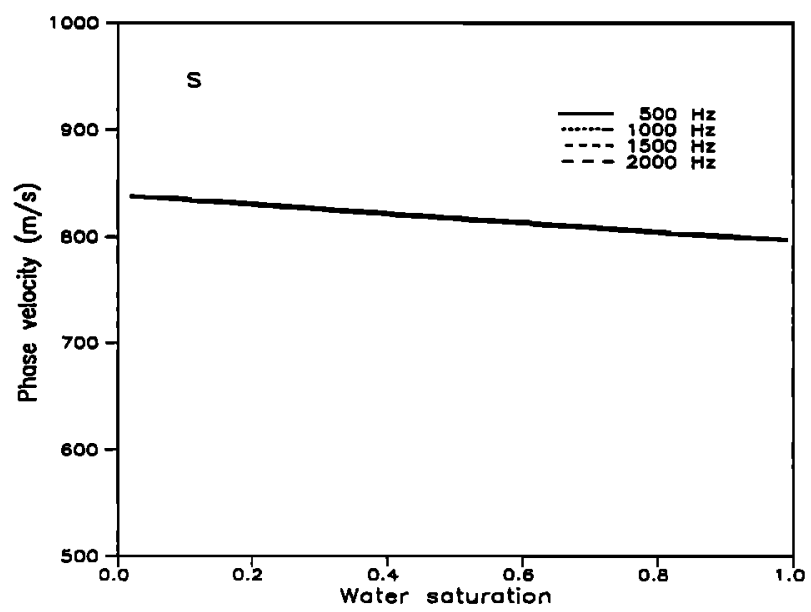

Figure 5. Effect of water saturation on the phase velocity of $\mathbf{S}$.

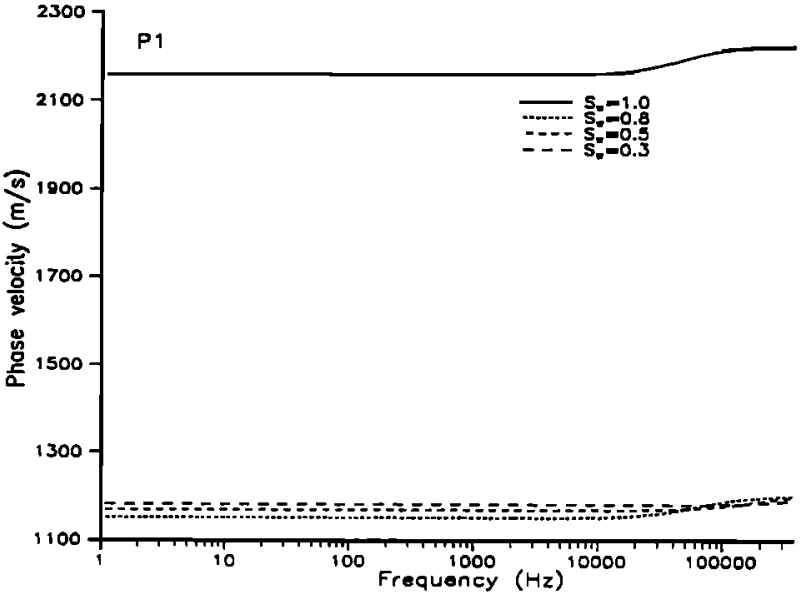

Figure 6. Frequency dependence of the phase velocity of P1.

than that of water which explains why P2 is much more attenuated in air-saturated samples than in water-saturated ones [Nagy et al., 1990; Nagy, 1993]. The attenuation coefficient of P3 is illustrated in Figure 12. As can be seen in Figure 12 , the attenuation coefficient becomes very large when the water saturation approaches zero or unity, that is, single pore fluid. Furthermore, the attenuation coefficient of $\mathrm{P} 3$ is always much larger than that of P1 and P2. Considering low velocity and high attenuation of $P 3$, we believe that an experimental observation of $\mathrm{P} 3$ is not possible. From the above discussion, we can conclude that $P 1$ is a wave, whereas $P 2$ and P3 are associated with a dissipative type of behavior. However, it should be noted that upon reflection and transmission of P1 at an interface, significant amounts of wave energy could be partitioned into $\mathbf{P 3}$ and dissipated into the medium under ideal circumstances. This would alter the amplitudes of the observable P1 waves and would not be accounted for in solid elasticity or even in Biot's poroelasticity theory.

We also observe that the presence of air reduces the phase velocity and increases the attenuation coefficient of $P 2$ which makes an experimental observation of P2 also difficult. Since Plona's [1980] first successful experiment, it is well known that water-saturated specimens have to be vacuum - impregnated to eliminate the remanent air saturation before the slow compressional wave $P 2$ appears. Similarly, air-saturated speci-

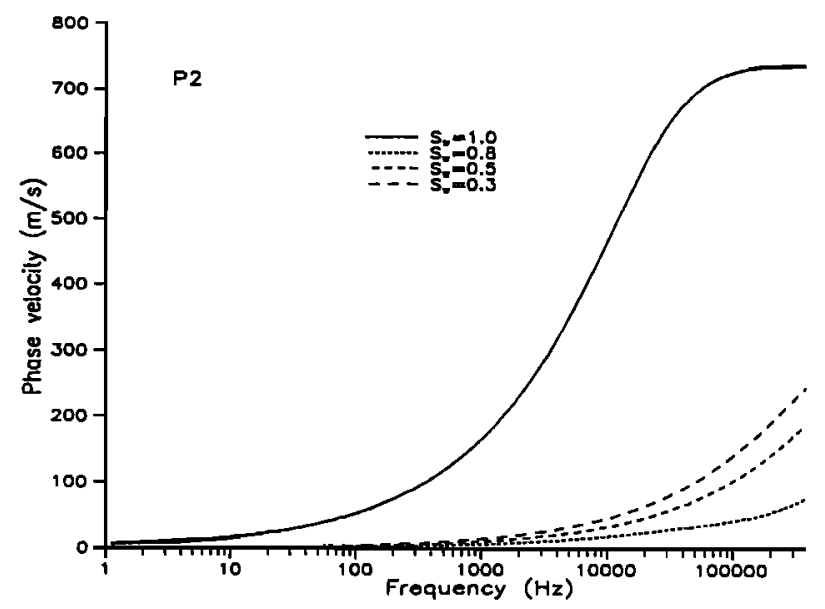

Figure 7. Frequency dependence of the phase velocity of $\mathbf{P} 2$. 


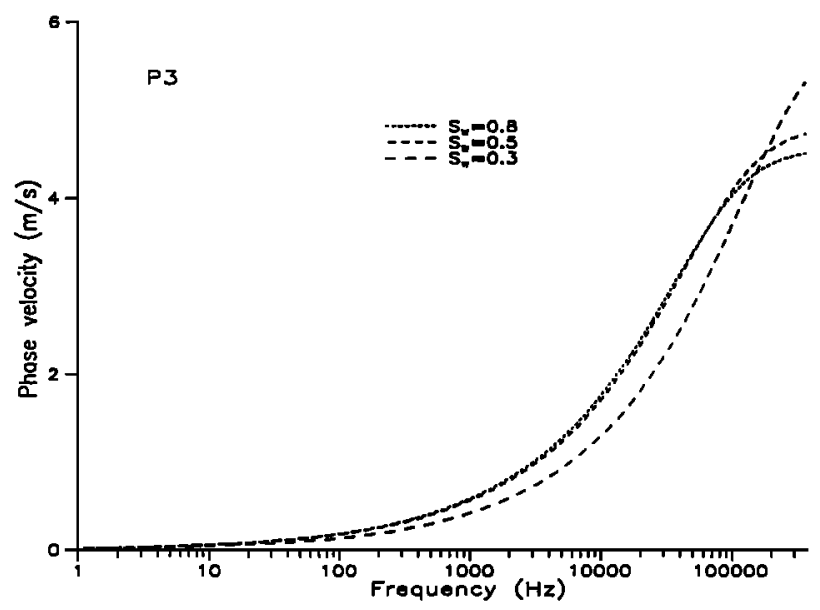

Figure 8. Frequency dependence of the phase velocity of P3.

mens have to be completely dried to remove the residual water saturation before P2 appears [Nagy et al., 1990]. Therefore as noted earlier, it seems to be highly probable that any degree of partial saturation eliminates P2. Figure 13 illustrates the attenuation coefficient of the rotational wave. Although the trend is similar to that of $\mathrm{Pl}$, we do not observe a peak in the attenuation coefficient. Comparing Figures 10 and 13, we find that attenuation coefficients of $P 1$ and $S$ are in the same order of magnitude.

\section{Comparison With Experimental Data}

Although there are several experimental studies in the literature on the phase velocities and attenuations of body waves in elastic porous media saturated by two fluids, most of them are limited to high-frequency wave propagation. Among the ones dealing with high-frequency wave propagation, one can note Domenico [1974, 1976], Gregory [1976, Elliot and Wiley [1975]. Domenico [1974, 1976] who investigated the effects of water saturation on reflection, refraction, and phase velocity of body waves, pointed out the difficulties in conducting experiments with multiphase fluids. Domenico [1974, 1976] demonstrated that the drainage process used in many laboratory studies results in heterogeneous gas distribution in

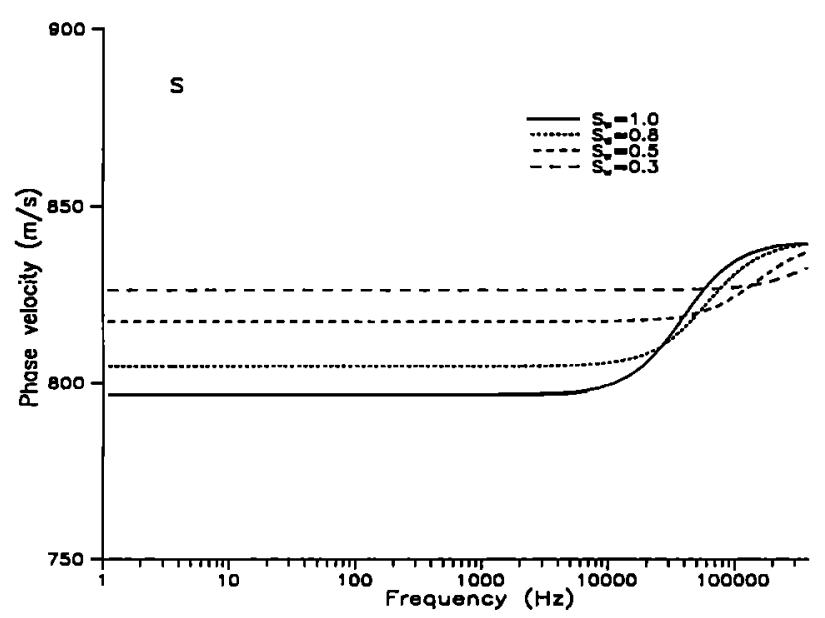

Figure 9. Frequency dependence of the phase velocity of $S$.

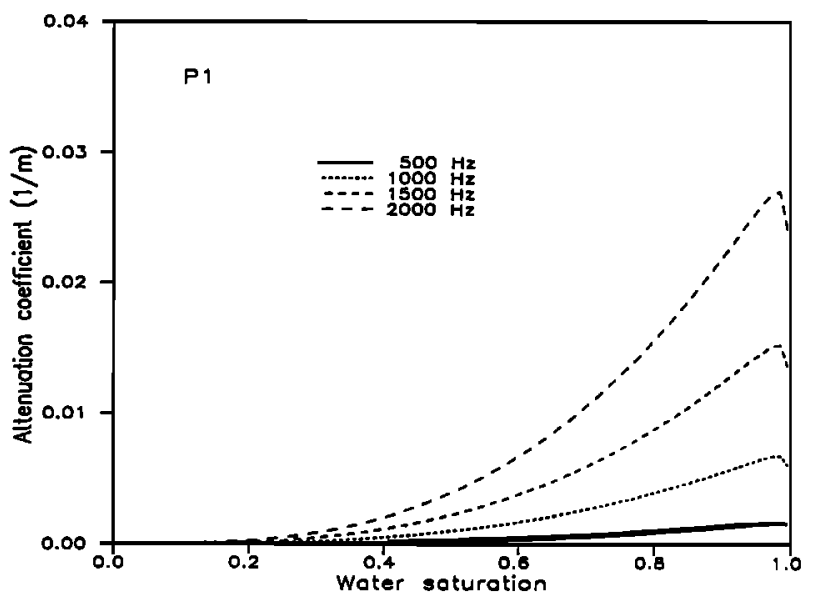

Figure 10. Effect of water saturation on the attenuation coefficient of P1.

the samples. Since we assume isotropy and homogeneity in the calculations, some of the discrepancies between our model results and the experimental data can be associated with Domenico's conclusions. Another comment was made by $Y$ in et al. [1992] who experimentally examined the effect of open and closed boundaries on the attenuation coefficient of P1. They found that the attenuation coefficient depends on saturation history as well as degree of saturation and boundary flow conditions. Yin et al. [1992] observed a significant difference in the attenuation of P1 during drainage and imbibition. Murphy [1982] provided experimental data on low-frequency wave attenuations and phase velocities in Massillon sandstone saturated by air and water. The material properties of Massillon sandstone are shown in Table 1. Parameters of (31) are chosen as $\alpha=0.025, n=10$, and $S_{12}=0.0$. We use the relative permeability curves of $W$ yckoff and Botset [1936] shown in Figure 1.

Figure 14 shows very favorable comparisons of model calculated and experimental phase velocities of P1 and S. As predicted by our model, experimental data show a jump in the phase velocity of $\mathrm{Pl}$ close to full water saturation. After a significant reduction, the phase velocity increases as the water saturation decreases. The phase velocity of $\mathrm{S}$ also increases as the water saturation decreases.

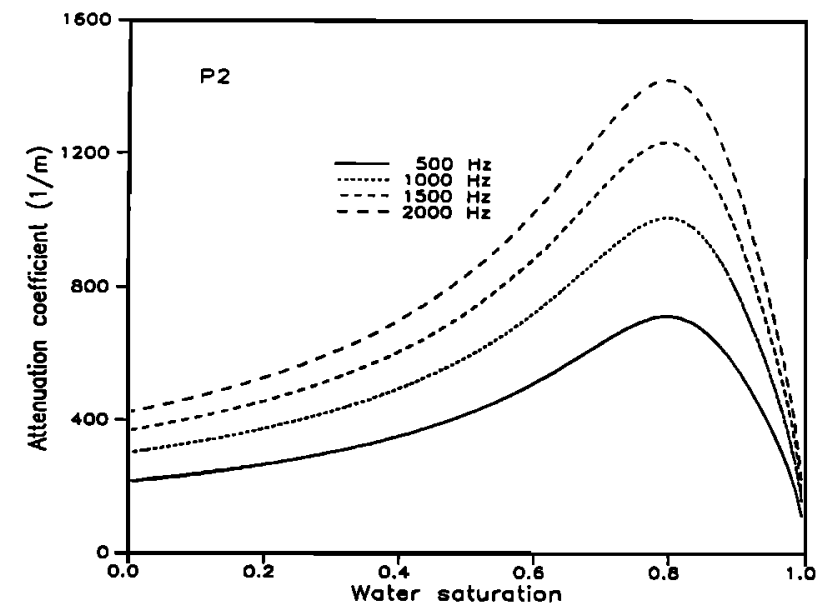

Figure 11. Effect of water saturation on the attenuation coefficient of $\mathbf{P} 2$. 


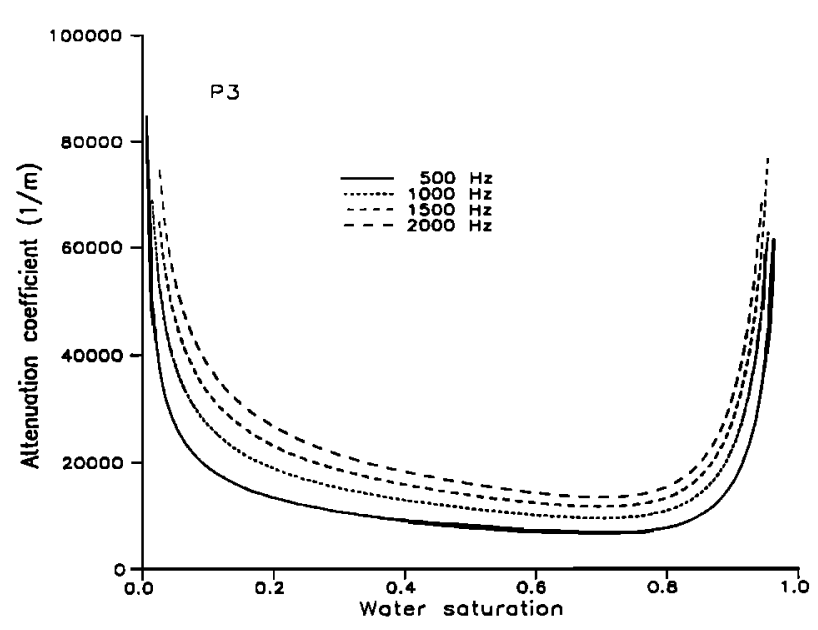

Figure 12. Effect of water saturation on the attenuation coefficient of $\mathbf{P 3}$.

As mentioned previously, our model considers the attenuation due to the relative movement of solid and fluid phases. Inelastic losses or contact relaxation are not taken into consideration. Thus we can only partially compare our results for the attenuation coefficients. Yin et al. [1992] observed an intrinsic attenuation at moderate water saturations, probably due to the moist rock frame which tends to behave like a standard inelastic solid. We inspect a similar trend of Murphy's [1982] experimental results. This part of the attenuation was analyzed by Winkler and Nur [1982], and Murphy et al. [1986]. As suggested by $Y$ in et al. [1992], we subtract this part of the attenuation to obtain the attenuation due to the relative movement of solid and fluid phases.

In Figures 15-16 we use the inverse quality factor, $1 / Q$, instead of the attenuation coefficient. Quality factor is a measure of the energy lost during one period of oscillation. The inverse quality factor is calculated from

$$
\frac{1}{Q}-\frac{2 c \xi_{i}}{\omega}
$$

Attenuation of $\mathrm{S}$ at a frequency of $500 \mathrm{~Hz}$ can be seen in Figure 15. The match is reasonable for water saturations less than 0.90 . Figure 16 shows the attenuation of $P 1$ as a function

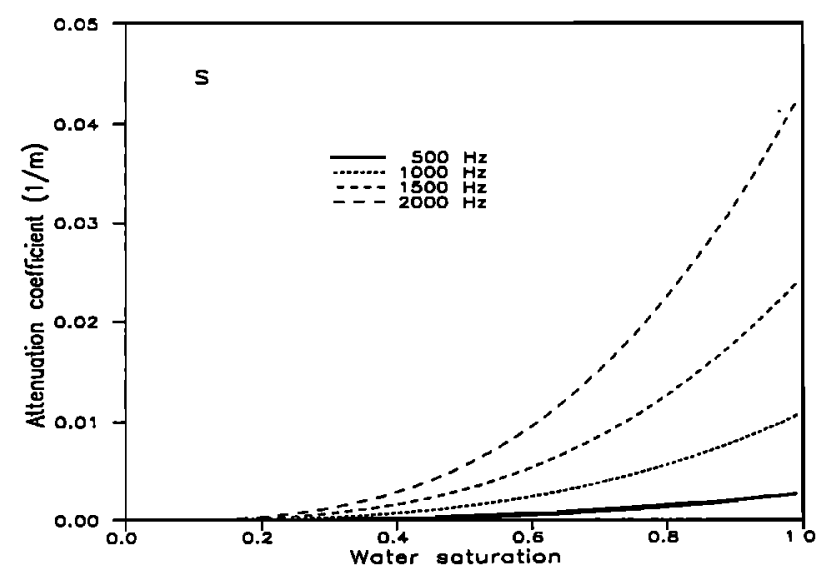

Figure 13. Effect of water saturation on the attenuation coefficient of $\mathrm{S}$.

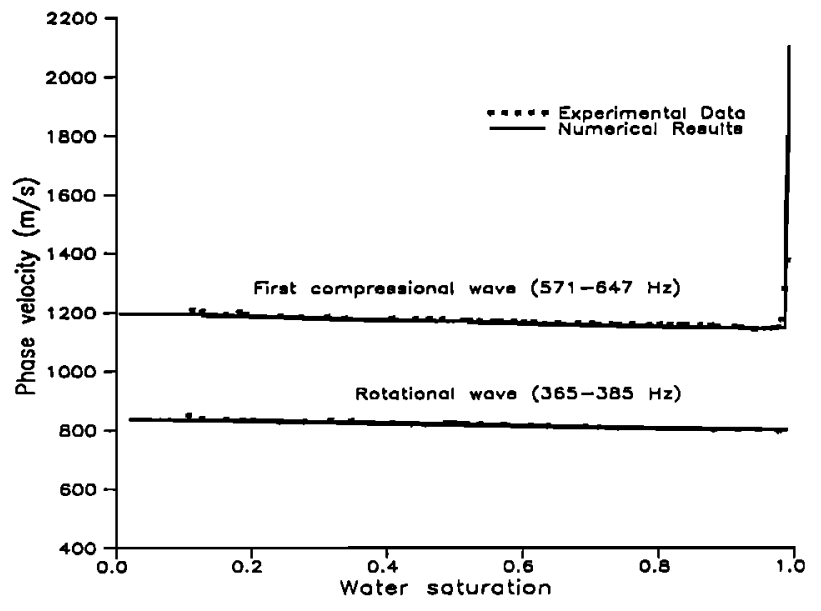

Figure 14. Comparison of predicted and experimental phase velocities of $P 1$ and $S$.

of water saturation for different values of permeability. Although both experimental and theoretical results exhibit a peak in the attenuation, the match is not favorable. As seen in Figure 16, attenuation of P1 at $600 \mathrm{~Hz}$ increases for larger permeabilities. Although permeability affects the magnitude of attenuation, the location of the peak of $1 / Q$ does not change.

We should note that although the experimental and theoretical peaks in the attenuation of $\mathrm{P} 1$ do not quite match, Yin et al. [1992] concluded that the peak is strongly dependent on the history of saturation. Their experimental results showed that attenuation peak for imbibition shifts toward a higher water saturation around $97 \%$.

\section{Conclusions}

We analyzed the characteristics of body waves in poroelastic porous media saturated by two immiscible Newtonian fluids. We demonstrated the existence of an additional compressional wave arising because of a second fluid phase in the pores. The first and second compressional waves are analogous to the fast and slow compressional waves in Biot's theory. We showed that the third compressional wave

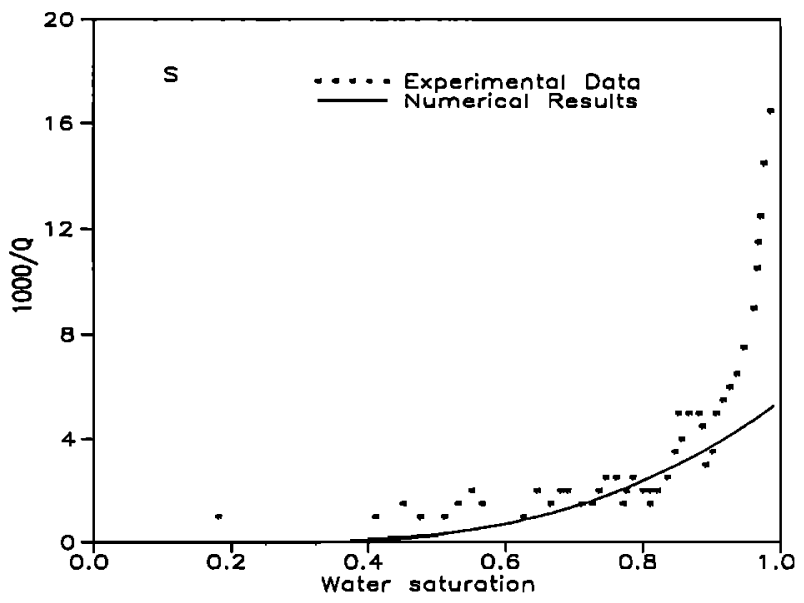

Figure 15. Comparison of predicted and experimental attenuation of $\mathrm{S}(500 \mathrm{~Hz})$. 


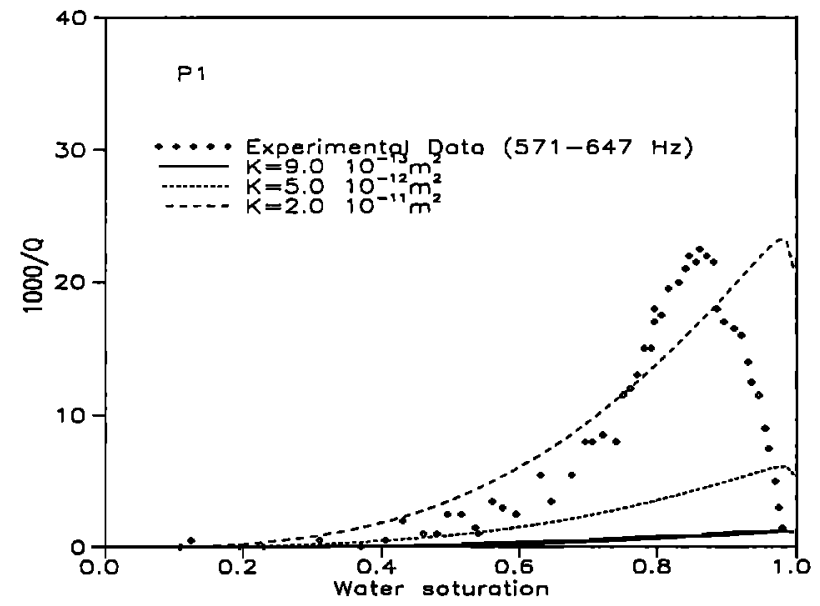

Figure 16. Effect of the intrinsic permeability on attenuation of P1 $(600 \mathrm{~Hz})$.

is associated with the pressure difference between the fluid phases and dependent on the slope of capillary pressuresaturation relation. We numerically examined the effect of water saturation and frequency on the phase velocities and attenuation coefficients of body waves for Massillon sandstone saturated by air and water phases. Our numerical results showed that presence of a gas phase significantly reduces the phase velocities of the first and second compressional waves. The third compressional wave has the lowest phase velocity and highest attenuation coefficient which make it very difficult to observe, if not impossible. We also examined the effect of water saturation on the attenuation coefficients. The attenuation coefficient of the first compressional wave is very small when the medium is saturated by air only. It increases as the water saturation increases and exhibits a peak at a very high water saturation. The attenuation of the rotational wave is similar except for the presence of a peak. We compared the results with the experimental data available in the literature. Although the phase velocity of the first compressional and rotational waves are well predicted by the theory, there is a discrepancy between the experimental and theoretical values of attenuation coefficients. However, it was previously shown that the drainage process used in many laboratory studies results in heterogeneous gas distribution in the samples [Domenico, 1974, 1976]. Since we assume isotropy and homogeneity in the calculations, some of the discrepancies between our model results and the experimental data can be associated with Domenico's conclusions. Yin et al. [1992] showed that the peak in the attenuation coefficient of P1 is strongly dependent on the history of saturation. Their experimental results demonstrated that attenuation peak for imbibition shifts toward a higher water saturation around $97 \%$. These comments may explain the discrepancies between the model results and experimental data for the attenuation coefficients.

\section{Appendix: Derivation of Governing Equations}

In this appendix we apply the volume averaging technique to obtain the governing equations of wave propagation in a linearly elastic porous medium saturated by two immiscible Newtonian fluids. The macroscale mass and momentum balance equations and constitutive relations will be obtained by volume averaging the corresponding microscale equations.
In the microscale, the grains will be assumed to be linearly elastic and the fluids are Newtonian. The coefficients of macroscopic constitutive relations will be expressed in terms of measurable quantities. Momentum transfer terms will be formulated in terms of intrinsic and relative permeabilities assuming the validity of Darcy's law.

\section{Microscopic Constitutive Relations, Mass and Momentum Balance Equations}

In this study the compressible porous medium consists of compressible solid grains and two immiscible viscous compressible fluids. Then the constitutive relations are given by

$$
\tau_{s}=K \boldsymbol{\nabla} \cdot u_{s} I+G_{s}\left(\nabla u_{s}+\left(\nabla u_{s}\right)^{T}-\frac{2}{3} \nabla \cdot u_{s} I\right)
$$

where $u_{s}, \tau_{s}, K_{s}, G_{s}$, and $I$ are the displacement, incremental stress tensor, bulk modulus, shear modulus of the solid phase, and the unit tensor, respectively. The superscript $T$ denotes the transpose of a tensor. We assume that both fluid phases are Newtonian with constitutive relations

$$
\tau_{i}=-P_{i} I+\mu_{i}\left(\nabla v_{i}+\left(\nabla v_{i}\right)^{T}-\frac{2}{3} \nabla \cdot v_{i} I\right) \quad j-1,2
$$

where $v_{i}, \tau_{i}, P_{i}$, and $\mu_{i}$ are the velocity, incremental stress tensor, incremental pore fluid pressure, and shear viscosity of fluid phase i, respectively. In (A2) the bulk viscosity of fluids is assumed to be negligible. The state equations of fluid phases are assumed to be in the form of

$$
\frac{1}{K_{i}} \frac{d P_{i}^{*}}{d t}=\frac{1}{\rho_{i}} \frac{d \rho_{i}}{d t} \quad i=1,2
$$

where $K_{i}, \rho_{i}$, and $P_{i}{ }^{*}$ are the bulk modulus, mass density, and pressure of phase $i$, respectively. The mass balance equations of fluid phases are expressed as

$$
\frac{1}{\rho_{i}} \frac{d \rho_{j}}{d t}=-\nabla \cdot v_{i} \quad i=1,2
$$

where $u_{i}$ is the displacement of the fluid phase $i$ from a reference position, that is, incremental displacement. We continue with the momentum balance equation in terms of incremental stresses and velocities as

$$
\nabla \tau_{j}=\rho_{j} \frac{d v_{j}}{d t} \quad j=s, 1,2
$$

We can rewrite (A5) by using the mass balance equations (A4) as

$$
\nabla \cdot \tau_{j}-\frac{\partial\left(\rho_{j} v_{j}\right)}{\partial t}+\nabla \cdot\left(\rho_{j} v_{j} v_{j}\right) \quad j-s, 1,2
$$

\section{Macroscopic Constitutive Relations}

Our next step is to obtain macroscopic constitutive relations by averaging the microscopic relations over a representative elementary volume [Bear, 1972]. Volume averaging of (A1) yields

$$
\begin{aligned}
& \frac{1}{V_{R_{s}}} \tau_{s} d V-K_{s}\left(\nabla \cdot\left(\alpha_{s} \bar{u}_{s}\right)+\frac{1}{V} \int_{S} u_{s} \cdot a d A\right) I \\
& +G_{s}\left(\nabla\left(\alpha_{s} \bar{u}_{s}\right)+\left(\nabla\left(\alpha_{s} \bar{u}_{s}\right)\right)^{T}-\frac{2}{3} \nabla \cdot\left(\alpha_{s} \bar{u}_{s}\right) I+K_{s i}\right) \quad i=1,2
\end{aligned}
$$


where

$$
K_{s i}=\frac{1}{V} \int_{S_{s i}}\left(u_{s} n_{s}+n_{s} u_{s}-\frac{2}{3} u_{s} \cdot n_{s} I\right) d A \quad i=1,2
$$

is a second-order tensor with zero trace. Since there is no mass exchange between the phases, the velocity of the interface is equal to the velocity of a point at the interface, that is, material surface. By employing the volume averaging rules, the integral in (A7) can be expressed as

$$
\frac{1}{V} \int_{S_{v i}} u_{s} \cdot n_{s} d A=\left(\alpha_{s}-\alpha_{s}^{0}\right)-\Delta \alpha_{s} \quad i=1,2
$$

where the superscript $(\theta)$ refers to the reference configuration. Since the displacements are assumed to be small, by definition $\bar{u}_{j} \cdot \nabla a_{j} \sim 0$. Then volume averaged constitutive relations for the solid phase can be expressed as

$$
\begin{aligned}
& \alpha_{s} \bar{\tau}_{s}-K_{s}\left(\alpha_{s} \nabla \cdot \overline{u_{s}}+\Delta \alpha_{s}\right) I \\
& \quad+G_{s}\left(\alpha_{s} \nabla \overline{u_{s}}+\alpha_{s}\left(\bar{\nabla} \bar{u}_{s}\right)^{T}-\frac{2}{3} \alpha_{s} \nabla \cdot \bar{u}_{s} I+K_{s j}\right) j=1,2
\end{aligned}
$$

where $\bar{\tau}_{\mathrm{s}}$ is the intrinsic averaged incremental stress of the solid phase. Similarly, the volume averaged constitutive relations for fluid phases are

$$
\begin{aligned}
& \alpha_{j} \bar{\tau}_{j}-K_{j}\left(\alpha_{i} \nabla \cdot \bar{u}_{i}+\Delta \alpha_{j}\right) I \\
& +\mu_{i}\left(\alpha_{j} \nabla \overline{v_{i}}+\alpha_{j}\left(\nabla \bar{v}_{j}\right) T_{-}-\frac{2}{3} \alpha_{j} \nabla \cdot \bar{v}_{i} I+J_{i j}+J_{i t}\right) i+k, \quad j-1,2 \\
& J_{i j}=\frac{1}{V} \int\left(v_{s_{i}} n_{i}+n_{j} v_{j}-\frac{2}{3} v_{j} \cdot n_{i} I\right) d A
\end{aligned}
$$

Under the small deformations assumption, the interfaces of the phases are not allowed to experience large deformations. Then by using Eqs. (A8) and (A12), and assuming $\partial u_{f} / \partial t>>$ $v_{i}, \nabla u_{i}$, we can write

$$
J_{i j}=\frac{\partial K_{i j}}{\partial t}
$$

We must note that here is a jump in the stresses of the immiscible fluids at the fluid-fluid interface because of the presence of interfacial tension and curvature of the interface. Assuming smooth pressure variations within the averaging volume, we can write

$$
\bar{P}_{1}^{*}-\bar{P}_{2}^{*}-P_{\text {cap }}\left(S_{1}\right)
$$

where $\overline{\mathbf{P}}_{1}^{*}$ and $\overline{\mathbf{P}}_{2}^{*}$ are the intrinsic averaged pressures. $\mathbf{P}_{\text {cap }}$, also known as capillary pressure is assumed to be a function of $S_{1}$ (saturation of the nonwetting phase) only. From now on fluid phase 1 will be considered as the nonwetting phase and fluid phase 2 as the wetting phase. $S_{1}$ is related to the volume fractions by

$$
S_{j}=\frac{\alpha_{i}}{1-\alpha_{s}} \quad j-1,2
$$

Then $S_{1}+S_{2}=1$. Noting that fluid pressures we work with are the incremental pressures, as a first-order approximation we can write

$$
\overline{P_{1}}-\overline{P_{2}}=\frac{d P_{\operatorname{cop}}}{d S_{1}} \Delta S_{1}
$$

provided that change in saturation, $\Delta S_{1}$ is small.
To explore the constitutive relations associated with the volume changes, we start by introducing $\bar{P}_{j}$

$$
-\alpha_{j} \bar{P}_{j}=\frac{1}{3} \operatorname{tr}\left(\boldsymbol{\alpha}_{j} \bar{\tau}_{j}\right)-K_{j}\left(\alpha_{j} \nabla \cdot \bar{u}_{j}+\Delta \alpha_{j}\right) \quad j-s, 1,2
$$

Equation (A17) does not contain any rotational deformations. For an elastic porous medium saturated by a single fluid, Pride et al. [1992] showed that

$$
\nabla \cdot \bar{u}_{s}=-\boldsymbol{\alpha}_{s} \frac{\left(\overline{P_{s}}-\bar{P}_{d}\right)}{K_{f r}}-\frac{\overline{P_{f}}}{K_{s}}
$$

where $K_{\mathrm{fr}}$ is defined as the "frame" or "drained" bulk modulus. We assume that in case of two fluids $\bar{P}_{\mathrm{f}}$ is given by

$$
\bar{P}_{f}-S_{1} \bar{P}_{1}+\left(1-S_{1}\right) \bar{P}_{2}
$$

Then, we can rewrite Eq. (A18) as

$$
\nabla \cdot \bar{u}_{s}=-a_{s} \frac{\left(\bar{P}_{s}-S_{1} \bar{P}_{1}-\left(1-S_{1}\right) \bar{P}_{2}\right)}{K_{f r}}-\frac{\left(S_{1} \bar{P}_{1}+\left(1-S_{1}\right) \bar{P}_{2}\right)}{K_{s}}
$$

We can express the change in volume fraction of the nonwetting phase from (A18) as

$$
\begin{aligned}
& \Delta \alpha_{1}-\Delta\left(S_{1}\left(1-\alpha_{s}\right)\right)-S_{1}\left(1-\alpha_{s}\right)-S_{1}^{0}\left(1-\alpha_{s}^{0}\right) \\
& \Delta \alpha_{1}-\left(1-\alpha_{s}\right) \Delta S_{1}-S_{1} \Delta \alpha_{s}
\end{aligned}
$$

and the change in volume fraction of the wetting phase as

$$
\begin{aligned}
& \Delta \alpha_{2}=\Delta\left(\left(1-S_{1}\right)\left(1-\alpha_{s}\right)\right)-\left(1-S_{1}\right)\left(1-\alpha_{s}\right)-\left(1-S_{1}^{0}\right)\left(1-\alpha_{s}^{0}\right) \\
& \Delta \alpha_{2}=-\left(1-\alpha_{s}\right) \Delta S_{1}-\left(1-S_{1}\right) \Delta \alpha_{s}
\end{aligned}
$$

Since we have already assumed that $\Delta S_{1}$ and $\Delta \alpha_{8}$ are small, the product of these terms can be neglected. Equations (A16), (A17), and (A20) can be rewritten in the matrix form, and solutions for the unknowns, $\Delta \alpha_{3}, \Delta S_{1}, \bar{P}_{8}, \bar{P}_{1}$, and $\bar{P}_{2}$ are obtained by inverting the coefficient matrix as

$$
\begin{gathered}
\Delta \alpha_{s}-b_{1} \nabla \cdot \bar{u}_{s}+b_{2} \nabla \cdot \bar{u}_{1}+b_{3} \nabla \cdot \bar{u}_{2} \\
\Delta S_{1}-c_{1} \nabla \cdot \bar{u}_{s}+c_{2} \nabla \cdot \bar{u}_{1}+c_{3} \nabla \cdot \bar{u}_{2} \\
-\alpha \bar{P}_{s}-a_{11} \nabla \cdot \bar{u}_{s}+a_{12} \nabla \cdot \bar{u}_{1}+a_{13} \nabla \cdot \bar{u}_{2} \\
-\left(1-\alpha_{s}\right) S_{1} \bar{P}_{1}=a_{21} \nabla \cdot \bar{u}_{s}+a_{22} \nabla \cdot \bar{u}_{1}+a_{23} \nabla \cdot \overline{u_{2}} \\
-\left(1-\alpha_{s}\right)\left(1-S_{1}\right) \bar{P}_{2}-a_{31} \nabla \cdot \bar{u}_{s}+a_{32} \nabla \cdot \bar{u}_{1}+a_{33} \nabla \cdot \overline{u_{2}}
\end{gathered}
$$

where $a_{i j}, b_{i}$, and $c_{i}$ are constants expressed in terms of material parameters (see (4)-(10)).

Our next step is the evaluation of the solid matrix's shear modulus $G_{\mathrm{rr}}$. We assume that all shear resistance of the porous medium is provided by the solid matrix only. This uncouples the shear deformation of all phases, that is, $K_{i j}=J_{i j}=0$. If an external shear stress $\tau_{e}$ is applied to the material, we can write

$$
\begin{aligned}
\tau_{e}-\alpha_{s} \overline{\tau_{s}}+\alpha_{1} \bar{\tau}_{1}{ }^{D}+\alpha_{2} \bar{\tau}_{2}{ }^{D} & \sim \alpha_{s} \bar{\tau}_{s} \\
& -G_{f r}\left(\nabla \overline{u_{s}}+\left(\bar{\nabla} \bar{u}_{s}\right)^{T}-\frac{2}{3} \nabla \cdot \bar{u}_{s} I\right)
\end{aligned}
$$

where $\tau_{j}{ }^{D}$ is the deviatoric stress of phase $j$. In other words, the fluids are viscous but the mechanical shear response of the 
porous medium is provided by the solid matrix only. Fluid viscosities will be taken into consideration later when we discuss the momentum transfers between the phases. We can rewrite the constitutive relations by introducing (A28) into (A25) and definitions of incremental stress tensor of phases 1 and 2 into (A26) and (A27).

$$
\begin{aligned}
& \left\langle\tau_{s}\right\rangle-\alpha_{s} \bar{\tau}_{s}-\left(a_{11} \nabla \cdot \bar{u}_{s}+a_{12} \nabla \cdot \overline{u_{1}}+a_{13} \nabla \cdot \overline{u_{3}}\right) I \\
& +G_{f r}\left(\overline{\nabla u_{s}}+\left(\nabla \overline{u_{s}}\right) T-\frac{2}{3} \nabla \cdot \bar{u}_{s} I\right) \\
& \left\langle\tau_{1}\right\rangle-S_{1}\left(1-\alpha_{s}\right) \overline{\tau_{1}}-\left(a_{21} \nabla \cdot \bar{u}_{s}+a_{22} \nabla \cdot \bar{u}_{1}+a_{23} \nabla \cdot \bar{u}_{3}\right) I \\
& \left\langle\tau_{2}\right\rangle-\left(1-S_{1}\right)\left(1-\alpha_{s}\right) \overline{\tau_{2}}-\left(a_{31} \nabla \cdot \bar{u}_{s}+a_{32} \nabla \cdot \overline{u_{1}}+a_{33} \nabla \cdot \overline{u_{3}}\right) I
\end{aligned}
$$

\section{Macroscopic Momentmm Balance Equations}

Employing the volume averaging theorems, the volume average of (A6) is obtained as

$$
\begin{aligned}
\frac{\partial\left\langle\rho_{j} V_{j}\right\rangle}{\partial t}+\nabla \cdot\left(\left\langle\rho_{j} V_{j} V_{j}\right\rangle\right)+\frac{1}{V_{S_{s}}} \int_{\rho_{j} V_{j}}\left(v_{j}-u\right) \cdot n_{j} d A \\
-\nabla \cdot\left\langle\tau \tau_{j}\right\rangle+\frac{1}{V_{S, j}} \int_{s_{j}} \tau_{j} \nabla_{j} d A \quad j \neq i, \quad j=s, 1,2
\end{aligned}
$$

The integral on the left-hand side of (A32) vanishes since the velocity of the interface is equal to the velocity of a point at the interface, that is, no mass exchange between the phases. We assume that the second term on the left-hand side can be neglected under the small deformation assumption. Average velocities and displacements for all phases are related by

$$
\left\langle v_{j}\right\rangle=\left\langle\frac{\partial u_{j}}{\partial t}\right\rangle-\frac{\partial\left\langle u_{j}\right\rangle}{\partial t}-\frac{1}{V} \int_{s_{i t}} u_{j} v_{j} \cdot n_{j} d A \quad j \neq i, \quad i=s, 1,2 \text { (A }
$$

Since we are interested in the low-frequency wave propagation, that is, characteristic length of the microscopic scale is smaller than the wavelength, the displacements appearing in the integrand in (A33) can be assumed to be constant. Employment of the averaging rules and substitution of (A33) in (A32) with linearization of $\left\langle p_{j}\right\rangle$ in the resulting equations yield

$$
\left\langle\boldsymbol{p}_{j}\right\rangle \frac{\partial^{2} \bar{u}_{j}}{\partial t}-\nabla \cdot\left\langle\tau_{j}\right\rangle+\frac{1}{V} \int_{S_{j}} \tau_{j} \cdot n_{j} d A \quad j \neq i, \quad j-s, 1,2
$$

\section{Momentum Transfer}

Due to the complexity of the pore-scale problem, we approximate the interaction terms by assuming the validity of Darcy's law. Since the theory is for low-frequency waves, the assumption of laminar flow is a reasonable one. Then,

$$
\begin{gathered}
\frac{1}{V} \int_{S_{s}} \tau_{s} \cdot n_{s} d A=\frac{\left(1-\alpha_{s}\right)^{2} S_{1}^{2} \mu_{1}}{K k_{r l}}\left(\overline{v_{1}}-\overline{v_{s}}\right) \\
\frac{1}{V} \int_{S_{s}} \tau_{s} \cdot n_{s} d A-\frac{\left(1-\alpha_{s}\right)^{2}\left(1-S_{1}\right)^{2} \mu_{2}}{K k_{r 2}}\left(\overline{v_{2}}-\overline{v_{s}}\right)
\end{gathered}
$$

where $\mathrm{K}$ is the intrinsic permeability of the porous medium and $k_{r i}$ is the relative permeability of phase $i$.
Substitution of the constitutive relations (equations (A29)(A31)) and the interaction terms (equations (A35) and (A36)) in the averaged momentum balance equations (A34) yield (1)(3). Equations (1)-(3) are the governing equations for lowfrequency wave propagation in a poroelastic medium saturated by two immiscible fluids with unknowns: $\bar{u}_{3}, \bar{u}_{1}$, and $\bar{u}_{2}$.

\section{References}

Bear, J., Dynamics of fuids in porous media, American Elsevier, New York, 1972.

Berryman, J.G., Confirmation of Biot's theory, Appl. Phys. Lett., 37, 382-384, 1980.

Biot, M.A., Theory of propagation of elastic wave in a fluid saturated porous solid, I, Low frequency range, J. Acoust. Soc. Am., 28, $168-178,1956 \mathrm{a}$.

Biot, M.A., Theory of propagation elastic waves in a fluid saturated porous solid, II, Higher frequency range, J. Acoust. Soc. Am., 28, 169-191, 1956b.

Brutsaert, W., The propagation of elastic waves in unconsolidated unsaturated granular mediums, J. Geophys. Res., 69, 243-257, 1964.

Brutsaert, W., and J.N. Luthin, The velocity of sound in soils near the surface as a function of the moisture content, $J$. Geophys. Res., 69 , 643-652, 1964.

Chattopadhyay, A., and R.K. De, Love wave types in a porous layer with irregular interface, Int. J. Eng. Sci, 21, 1295-1303, 1983.

Corapcioglu, M.Y., and K. Tuncay, Propagation of waves in porous media, in Advances in Porous Media, 3, edited by M.Y. Corapcioglu, 361-440, Elsevier, Amsterdam, 1996.

Deresiewicz, $H$., The effect of boundaries on wave propagation in a liquid-filled porous solid, II, Love waves in a porous layer, Bull. Seiomol. Soc. Am, 5I, 51-59, 1961.

Deresiewicz, $H$., The effect of boundaries on wave propagation in a liquid-filled porous solid, IV, Surface waves in a baif-space, Bull. Scismol. Soc. Am., 52, 627-638, 1962.

Deresiewicz, H., The effect of boundaries on wave propagation in a liquid-filled porous solid, VI, Love waves in a double surface layer, Bull. Seiomol. Soc. Am., 54, 417-423, 1964

Deresiewicz, $H$., The effect of boundaries on wave propagation in a liquid-filled porous solid, $\mathbf{X}$, Love waves in a porous internal stratum, Bull. Seisnol. Soc. Am., 55, 919-923, 1965.

Domenico, S.N., Effects of water saturation of sand reservoirs encased in shales, Geophysics, 29, 759-769, 1974.

Domenico, S.N., Effect of brine-gas mixture on velocity in an unconsolidated sand reservoir, Geophysias 41, 882-894, 1976.

Elliott, S.E., and B.F. Wiley, Compressional velocities of partially saturated, unconsolidatod sands, Geophysics, 40, 949-954, 1975.

Garg, S.K., and A.H. Nayfeh, Compressional wave propagation in liquid and/or gas saturated elastic porous media, J. Appl. Phys., 60, 3045-3055, 1986.

Gregory, A.R., Fluid saturation effocts on dynamic elastic properties of sedimentary rocks, Geophysica, 41, 895-921, 1976.

Jones, J.P., Rayleigh waves in a porous, elastic, saturated solid, $J$. Acoust. Soc. Am., 33, 959-962, 1961.

Miksis, M.,J., Effects of contact line movement on the dissipation of waves in partially saturated rocks, J. Geophys. Res, 93, 6624-6634, 1988.

Mochizuki, S., Attenuation in partially saturated rocks, $J$. Geophys. Res., 87, 8598-8604, 1982.

Murphy, W.F., Effects of partial water saturation on attenuation in Massillon sandstone and Vycor porous glass, J. Acoust. Soc. Am., 71, 1458-1468, 1982.

Murphy, W.F., Acoustic measures of partial gas saturation in tight sandstones, J. Geophys. Res., 89, 11549-11559, 1984.

Murphy, W.F., K.W. Winkler, and R.L. Kleinberg, Acoustic relaxation in sedimendary rocks: Dependence on grain contacts and fluid saturation, Geophysics, 51, 757-766, 1986.

Nagy, P. B., L. Adler, and P.B. Bonner, Slow wave propagation in airfilled porous materials and natural rocks, Appl. Phys. Lett., 56, 2504-2509, 1990.

Nagy, P. B., Slow wave propagation in air-filled permeable solids, $J$. Acoust. Soc. Am., 9.3, 3224-3234, 1993.

Plona, T.J., Observation of a second bulk compressional wave in a porous medium at ultrasonic frequencies, Appl. Phys. Lett., 36, 259-261, 1980. 
Pride, S.R., A.F. Gangi, and F.D. Morgan, Deriving the equations of motion for isotropic media, J. Acoust. Soc. Am., 88, 3278-3290, 1992.

Santos, J.E., J.M. Corbero, and J. Douglas, Static and dynamic behavior of a porous solid, J. Acoust. Soc. Am., 87, 1428-1438, 1990.

Tajuddin, M., Rayleigh waves in a poroelastic half-space, J. Acoust. Soc. Am., 75, 682-684, 1984.

Tuncay, K., Wave propagation in single- and double-porosity deformable porous media saturated by multiphase fluids, $\mathrm{Ph} . \mathrm{D}$ Dissertation, Tex. A\&M Univ., College Station, 1995.

Van Genuchten, M. T., A closed form equation for prodicting the hydraulic conductivity of unsaturated soils. Soil Sci. Soc. Am. J., 44, 892-898, 1980.
Winkler, K.W., and A. Nur, Seismic attenuation: Effect of pore fluids and frictional sliding, Geophysics 47, 1-15, 1982.

Wyckoff, R.D., and H.G. Botset, The flow of gas-liquid mixture through unconsolidated sands, Physias, 7, 325-345, 1936.

Yin, C.S., M.I. Batzle, and B.J. Smith, Effects of partial liquid/gas saturation on extensional wave attenuation in berea sadstone, Geophys. Res. Lett., 19, 1399-1402, 1992.

M. Yavuz Corapcioglu, Department of Civil Engineering, Texas A\&M University, College Station, TX 77843-3136.

K. Tuncay, Faculty of Engineering, Izmir Institute of Technology, Gaziosmanpasa Bulvari, No.16, Cankaya, Izmir, Turkey.

(Received March 9, 1995; revised July 1, 1996; accocpted July 11, 1996.) 